\title{
Intentional weight loss as a predictor of type 2 diabetes occurrence in a general population
}

\section{Abstract}

Obesity is a major risk factor of type 2 diabetes (T2D). Accordingly, weight loss is being used to reduce this risk. Intervention studies have shown a T2D risk reduction due to weight loss in populations with an elevated risk. Data on the prediction of intentional weight loss (IWL) on diabetes occurrence at population level is, however, sparse. Since in observational studies IWL has been shown to predict later weight gain, possibly leading to adverse consequences, this study examined the prediction of IWL on T2D occurrence in a general population. Data from the representative Finnish Health 2000 Survey conducted in 2000-2001 was used. The study sample consisted of 1962 men and 2308 women, aged 30-69. Information on IWL during the previous year was collected with a questionnaire. Data for the covariates was collected with a health examination, interviews and questionnaires. Incident T2D cases during a 15-year follow-up were drawn from national health registers covering information on medication use, hospitalization, and cause of death. The Cox model was used to estimate strength of association between IWL and T2D occurrence. During the follow-up, 417 individuals developed a T2D. After adjusting for socio-demographic and lifestyle factors and the components of metabolic syndrome, IWL predicted an increased risk of T2D occurrence (HR 1.44; 95\% CI 1.11-1.87) compared to individuals without IWL. Moreover, in interaction analyses, the risk in individuals with IWL compared to those without IWL was pronounced in younger age group and in those with the lowest quality diet. Furthermore, an elevated risk was consistently found in persons with IWL and overweight (BMI $\geq$ $25 \mathrm{~kg} / \mathrm{m} 2$ ), also in combination with different aspects of poor health (i.e. mental health, self-perceived health, metabolic health). In the present cohort, IWL predicted an increased subsequent risk of developing T2D. This may be due to that in long-term at population level IWL causes an elevated risk of T2D e.g. by acting as a promotor for weight gain or by causing weight cycling resulting in unfavorable changes in metabolic health indicators. Alternatively, it cannot be excluded, that individuals reporting IWL already are at a greater risk of T2D and pursue IWL in response to that. Greater emphasis should be put on the initial prevention of weight gain and on the support on the weight maintenance after weight loss. Further cohort studies with repeated measurements on dieting behavior and changes in weight are needed.

\section{Conflict of Interest}

There is no conflict of interest 